\title{
Recent results on glucose-insulin predictions by means of a state observer for time-delay systems
}

\author{
Pasquale Palumbo, Pierdomenico Pepe, Simona Panunzi, Andrea De Gaetano
}

\begin{abstract}
To achieve accurate and affordable predictions of glucose and insulin plasma concentrations is of paramount importance, especially in the field of the artificial pancreas, where real-time measurements could be properly exploited in model-based glucose control algorithms. This note focuses on a recently developed research line that makes use of a state observer to estimate insulin in real-time from glucose measurements, since it is known that insulin measurements are slower and more cumbersome to obtain, more expensive and also less accurate. Based on these predictions, glucose control algorithms can be designed, and can be exploited for both intra-venous and subcutaneous insulin infusions. The safety, robustness and efficacy of the observer-based control algorithms have been validated on a population of rather heterogenous virtual patients, modeled by a different, comprehensive model of the glucose-insulin system, recently accepted by the Food and Drug Administration as a substitute of animal trials.
\end{abstract}

\section{Introduction}

Diabetes Mellitus (DM) is a worldwide disease with an alarming increase, especially in the developing countries: the diabetic population, estimated to be around 171 million people in 2000, has been predicted to double within 2030, [38]. To diagnose and contain the spread of DM disease, glucose and insulin predictions are of great importance, especially when required in closed-loop, real-time algorithms for

P. Palumbo, S. Panunzi, A. De Gaetano

BioMatLab, IASI "A. Ruberti", National Council of Researches, UCSC Largo A. Gemelli 8, 00168 Rome, e-mail: pasquale.palumbo@iasi.cnr.it, simona.panunzi@biomatematica.it, andrea.degaetano@biomatematica.it

P. Pepe

Department of Information Engineering, Computer Science and Mathematics, University of L'Aquila, Via Gronchi 18,67100 L'Aquila e-mail: pierdomenico.pepe@univaq.it 
the artificial pancreas. Differently from plasma glycemia, which can be straightforwardly measured with relative low-cost devices and affordable algorithms, plasma insulinemia is slower and more cumbersome to obtain, more expensive and also less accurate. This fact stimulates the study of algorithms capable of providing the plasma insulin concentration by processing a stream of glycemia measurements. When these algorithms exploit the available measurements as coming from a known deterministic dynamical model generating them, we deal with state observers. The importance of these powerful tools is due to the great variety of observer-based control law applicable, at least in theory, to the glucose control problem, with exogenous insulin administration playing the role of the control input. Observer-based closed-loop control laws belong to the field of model-based strategies, that means the regulator is synthesized by explicitly exploiting the structure of the model equations [4].

Differently from the great majority of model-based approaches, which use nonlinear Ordinary Differential Equation (ODE) models, a nonlinear discrete-Delay Differential Equation (DDE) model of the glucose/insulin system is considered $[20,32]$. Motivation to use DDE models is that they provide a better representation of the pancreatic Insulin Delivery Rate (IDR) (e.g., [17, 12] and references therein), therefore allowing to treat in a unified fashion both Type 1 and Type 2 diabetic patients, these latter with a not negligible IDR. More in details, the adopted glucose/insulin DDE model has been shown to exhibit a number of desirable characteristics, such as to conform to established physiological concepts (e.g., pancreatic insulin secretion rate is limited), to exhibit satisfactory properties of the solutions (e.g., positivity and boundedness of solutions, local attractivity of a single positive equilibrium, [20]), and to be statistically robust, in that its parameters are identifiable with very good precision, fitting the model onto observations from standard perturbation experiments, such as the Intra-Venous Glucose Tolerance Test (IVGTT), $[32,31]$.

Different results are here reported, dealing with theoretical design (feedback linearization with delay cancelation, state observers, asymptotic convergence, local Input-to-State Stability) and practical issues (discretization of the control algorithm, measurement uncertainties, insulin pump malfunctioning, intra-patient variability) according to both intravenous $[22,23,25,26]$ and subcutaneous $[24,21,28]$ insulin delivery. Although the proposed control laws require the knowledge of the whole state of the system (i.e. both glycemia and insulinemia, possibly also at retarded instants) only glucose measurements have been exploited, leaving the task to provide real-time insulin estimates to an observer for nonlinear time-delay systems. Safety, efficacy and robustness have been recently validated [30] on a population of Virtual Patients (VP) generated by a different, comprehensive model of the glucose-insulin system, recently accepted by the Food and Drug Administration as a substitute of animal trials, [15].

The chapter continues as follows. Next Section is devoted to introduce the DDE model of the glucose-insulin system adopted for the state prediction task and, consequently, for the synthesis of the closed-loop control. Sections 3 and 4 deal with 
the intravenous and subcutaneous insulin delivery modes, detailing with the most important results achieved. Conclusions follow.

\section{The DDE model of the glucose-insulin system}

The model-based algorithms here reported for the artificial pancreas have been synthesized according to the following DDE model of the glucose-insulin system [20, 32]. Symbols 'mmol' and 'pmol' stand for millimoles and picomoles, with ' $\mathrm{mM}$ ' and ' $\mathrm{pM}$ ' denoting mmoles/liter and pmol/liter, respectively. The equations are written with respect to plasma glycemia, $G(t),[\mathrm{mM}]$, and insulinemia, $I(t)$, $[\mathrm{pM}]:$

$$
\begin{aligned}
& \frac{d G(t)}{d t}=-K_{x g i} G(t) I(t)+\frac{T_{g h}}{V_{G}}+\frac{d(t)}{V_{G}}, \\
& \frac{d I(t)}{d t}=-K_{x i} I(t)+\frac{T_{i G \max }}{V_{I}} f\left(G\left(t-\tau_{g}\right)\right)+\frac{u(t)}{V_{I}},
\end{aligned}
$$

with $K_{x g i},\left[\min ^{-1} \mathrm{pM}^{-1}\right]$, the rate of (insulin-dependent) glucose uptake by tissues per $\mathrm{pM}$ of plasma insulin concentration; $T_{g h},[(\mathrm{mmol} / \mathrm{kgBW}) / \mathrm{min}]$, the net balance between hepatic glucose output and insulin-independent zero-order glucose tissue uptake; $V_{G}, V_{I},[\mathrm{~L} / \mathrm{kgBW}]$, the apparent distribution volumes for glucose and insulin, respectively; $K_{x i},\left[\mathrm{~min}^{-1}\right]$, the apparent first-order disappearance rate constant for insulin; $T_{i G \max },[(\mathrm{pmol} / \mathrm{kgBW}) / \mathrm{min}]$, the maximum rate of second-phase insulin release; $\tau_{g}$, [min], the apparent delay with which the pancreas varies secondary insulin release in response to varying plasma glucose concentrations.

The following Hill function is chosen for the nonlinear map $f(\cdot)$, modeling the endogenous pancreatic insulin delivery rate:

$$
f(G)=\frac{\left(G / G^{*}\right)^{\gamma}}{1+\left(G / G^{*}\right)^{\gamma}},
$$

with $\gamma$ the Hill coefficient and $G^{*}$, $[\mathrm{mM}]$, the glycemia at which the insulin release is half of its maximum rate.

The exogenous signals $u(t)$, [( $\mathrm{pmol} / \mathrm{kgBW}) / \mathrm{min}]$, and $d(t),[(\mathrm{mmol} / \mathrm{kgBW}) / \mathrm{min}]$, are the control input (i.e. the external insulin delivery rate) and a disturbance in the glucose dynamics (e.g. the glucose intake from a meal). As a matter of fact, model (1) does not consider the subcutaneous depot assuming that insulin is straightforwardly delivered intravenously. On the other hand, in case of subcutaneous insulin administration, equations (1) are modified as follows:

$$
\begin{aligned}
& \frac{d G}{d t}=-K_{x g i} G(t) I(t)+\frac{T_{g h}}{V_{G}}+\frac{d(t)}{V_{G}}, \\
& \frac{d I}{d t}=-K_{x i} I(t)+\frac{T_{i G \max }}{V_{I}} f\left(G\left(t-\tau_{g}\right)\right)+\frac{S_{2}(t)}{V_{I} t_{\max , I}},
\end{aligned}
$$




$$
\begin{aligned}
\frac{d S_{2}}{d t} & =\frac{1}{t_{\max , I}} S_{1}(t)-\frac{1}{t_{\max , I}} S_{2}(t), \\
\frac{d S_{1}}{d t} & =-\frac{1}{t_{\max , I}} S_{1}(t)+u(t),
\end{aligned}
$$

with $S_{1}, S_{2}$ [pmol/kgBW] the insulin mass in the accessible and not-accessible subcutaneous depot, respectively, and $t_{\max , I},[\mathrm{~min}]$, the time-to-maximum insulin absorption. In this framework the control input $u(t)$, [pmol/ $/ \mathrm{kgBW} / \mathrm{min}]$, is the exogenous insulin infusion rate, delivered subcutaneously. The model of insulin absorption here adopted (third and fourth equations in (3)) refers to [35] with no insulin degradation at the injection site. It has been recently analyzed in [39], and it has been exploited with the aim of glucose control in [11], according to which, here we assume the same notation.

As far as the initial conditions, the subject is supposed to be at rest before the insulin therapy starts, so that plasma glycemia and insulinemia are equal to the constant (hyperglycemic for uncontrolled diabetic patients) basal levels $\left(G_{b}, I_{b}\right)$ :

$$
G(\tau)=G_{b}, \quad I(\tau)=I_{b}, \quad \tau \in\left[-\tau_{g}, 0\right] .
$$

In case of model (3), besides (4) we have that the subcutaneous depots are empty, therefore:

$$
S_{1}(\tau)=0, \quad S_{2}(\tau)=0, \quad \tau \in\left[-\tau_{g}, 0\right] .
$$

It has to be stressed that models (1-3) may represent equally well healthy subjects and insulin-resistant or severely insulin-deficient diabetic patients, by appropriately changing the parameter values. Moreover, it does belong to the class of "minimal models", in the sense that according to a "minimal" set of independent parameters, it allows to very well resemble the physiology of the glucose/insulin kinetics, and it is identifiable from data with very good precision, according to IVGTT standard perturbation experiments (see [32,31]).

\section{Observer-based control by means of intravenous insulin infusion}

Exogenous insulin administration is the basic procedure to cope with diabetes: for Type 1 patients only exogenous insulin is available, while for Type 2 exogenous insulin complements pancreatic production. The use of intravenous insulin administration, delivered by automatic, variable speed pumps under the direct supervision of a physician, provides a wide range of possible strategies and ensures a rapid delivery with negligible delays. As a matter of fact, control algorithms based on intravenous infusions (we can cite, among the others, [33, 36, 5, 7, 14, 13, 25]) are directly applicable so far only to problems of glycemia stabilization in critically ill subjects, such as in surgical Intensive Care Units after major procedures, [37]. 
The aim of the proposed control law therapies is to reduce a high basal plasma glucose concentration to a lower level, according to a smooth reference glucose trajectory $G_{\text {ref }}(t)$. The equations of model (1) will be taken into account. The control law is synthesized disregarding any disturbances in the glucose dynamics, like meals. The ability of the controller to reject these disturbances will play a crucial role in the evaluation of the artificial pancreas.

\subsection{Synthesis of the glucose control law}

Define the tracking error as follows:

$$
e(t)=\left[\begin{array}{l}
e_{1}(t) \\
e_{2}(t)
\end{array}\right]=Z(t)-Z_{\mathrm{ref}}(t)
$$

with:

$$
Z(t)=\left[\begin{array}{c}
z_{1}(t) \\
z_{2}(t)
\end{array}\right]=\left[\begin{array}{c}
G(t) \\
-K_{x g i} G(t) I(t)+\frac{T_{g h}}{V_{G}}
\end{array}\right], \quad Z_{\mathrm{ref}}(t)=\left[\begin{array}{c}
G_{\mathrm{ref}}(t) \\
\dot{G}_{\mathrm{ref}}(t)
\end{array}\right],
$$

where $G_{\text {ref }}(t)$ is the glucose reference signal to be tracked. It is supposed to be bounded (with lower bound strictly positive), twice continuously differentiable, with bounded first and second derivatives. Since $G_{\text {ref }}(t)$ provides the desired plasma glucose concentration, it clearly comes that these constraints readily match with a physiologically meaningful choice. Examples of suitable $G_{\text {ref }}(t)$ can be found in $[22,25,30]$, where a function exponentially decreasing from the hyperglycemic basal state down to a safe euglycemic level is chosen.

In [22], according to the theory of input-output feedback linearization with delay cancellation (see $[8,10,19]$ ), with respect to the output $y(t)=G(t)$ and the input $u(t)$, it is shown that, by applying the control input

$$
\frac{u(t)}{V_{I}}=\frac{S\left(G(t), I(t), G\left(t-\tau_{g}\right)\right)-v(t)}{K_{x g i} G(t)}, \quad t \geq 0,
$$

with

$$
\begin{array}{r}
S\left(G(t), I(t), G\left(t-\tau_{g}\right)\right)=-K_{x g i} I(t)\left(-K_{x g i} I(t) G(t)+\frac{T_{g h}}{V_{G}}\right) \\
-K_{x g i} G(t)\left(-K_{x i} I(t)+\frac{T_{i G \max }}{V_{I}} f\left(G\left(t-\tau_{g}\right)\right)\right)
\end{array}
$$

and

$$
v(t)=\ddot{G}_{\text {ref }}(t)+R e(t),
$$

the tracking error dynamics can be written as: 


$$
\dot{e}(t)=H e(t), \quad H=\left[\begin{array}{ll}
0 & 1 \\
0 & 0
\end{array}\right]+\left[\begin{array}{l}
0 \\
1
\end{array}\right] R .
$$

Because of the structure of $H$, the control matrix $R \in \mathbb{R}^{1 \times 2}$ can be designed such that matrix $H$ has pescribed eigenvalues in the left half complex plane, thus ensuring the exponential convergence to zero of the tracking error, that implies $G(t) \mapsto G_{\text {ref }}(t)$.

Such a control law (8-10) requires both glucose and insulin measurements: on the other hand, insulin measurements are slower and more cumbersome to obtain, more expensive, and also less accurate than glucose measurements: a need exists, therefore, to construct a control law avoiding the measurements of insulin serum. For these reasons, a state observer for system (1) has been considered in [25], in order to estimate the plasma insulin concentration and design a feedback control law based on only glucose measurements. To this end, denote with $\widehat{G}(t), \widehat{I}(t)$ the glucose and insulin estimates and consider the following equations for the observer:

$$
\begin{aligned}
& {\left[\begin{array}{c}
d \widehat{G} / d t \\
d \widehat{I} / d t
\end{array}\right]=\left[\begin{array}{c}
-K_{x g i} \widehat{G}(t) \widehat{I}(t)+\frac{T_{g h}}{V_{G}} \\
-K_{x i} \widehat{I}(t)+\frac{T_{i G \max }}{V_{I}} f\left(\widehat{G}\left(t-\tau_{g}\right)\right)+\frac{u(t)}{V_{I}}
\end{array}\right]} \\
& +Q^{-1}(\widehat{G}(t), \widehat{I}(t)) W(G(t)-\widehat{G}(t)),
\end{aligned}
$$

where $Q^{-1}$ is the inverse matrix of the matrix function $Q\left(x_{1}, x_{2}\right) \in \mathbb{R}^{2 \times 2}$ defined as:

$$
Q\left(x_{1}, x_{2}\right)=\left[\begin{array}{cc}
1 & 0 \\
-K_{x g i} x_{2} & -K_{x g i} x_{1}
\end{array}\right]
$$

and the observer gain matrix $W \in \mathbb{R}^{2 \times 1}$ is designed to ensure that

$$
\widehat{H}=\left[\begin{array}{ll}
0 & 1 \\
0 & 0
\end{array}\right]-W\left[\begin{array}{ll}
1 & 0
\end{array}\right]
$$

is Hurwitz with prescribed eigenvalues in the left half complex plane. The initial conditions for (12) are formally given by:

$$
\widehat{G}(\tau)=\widehat{G}_{0}(\tau), \quad \widehat{I}(\tau)=\widehat{I}_{0}(\tau), \quad \tau \in\left[-\tau_{g}, 0\right] .
$$

According to [25] and references therein, it comes that the observer can be designed such that, if the estimation error at zero is sufficiently small and the input signal is suitably bounded, the estimation error converges exponentially to zero, with arbitrary decay rate fixed by means of a suitable choice of $W$. Moreover, substitute the real glucose and insulin values in (8-9) with the estimates coming from the observer:

$$
\frac{u(t)}{V_{I}}=\frac{S\left(\widehat{G}(t), \widehat{I}(t), \widehat{G}\left(t-\tau_{g}\right)\right)-v(t)}{K_{x g i} \widehat{G}(t)}, \quad t \geq 0,
$$

and consider

$$
v(t)=\ddot{G}_{\mathrm{ref}}(t)+\operatorname{Re}(t)
$$


with the target error $\hat{e}(t)$ defined by $\hat{e}(t)=\widehat{Z}(t)-Z_{\text {ref }}(t)$, with:

$$
\widehat{Z}(t)=\left[\begin{array}{c}
\hat{z}_{1}(t) \\
\hat{z}_{2}(t)
\end{array}\right]=\left[\begin{array}{c}
\widehat{G}(t) \\
-K_{x g i} \widehat{G}(t) \widehat{I}(t)+\frac{T_{g h}}{V_{G}}
\end{array}\right] .
$$

It is proven in [25] that there exist gain matrices $R, W$ such that the closed-loop system given by (1) with $d(t)=0,(12),(16-17)$ ensures that the plasma glycemia is controlled to track the reference trajectory, with the error tracking asymptotically converging to zero, provided that the initial tracking and observer errors are suitably small. It worths noticing that such a result is ensured by exploiting both the estimated glucose and insulin concentrations in the control law. To use the real (and available) glucose measurements, instead of the estimated glycemia, would not ensure improvements nor (and more important) would it ensure to maintain the aforementioned theoretical results.

Remark 1 . Since the theory does not explicitly take into account the impossibility to release negative insulin, in this cases the regulator would temporarily switch off, leaving the patient without control for an unpredictable period. This is clearly an undesirable situation, to be avoided. Another important requirement is to prevent glucose oscillations, possibly determining dangerous hypoglycemia. Both these issues need to be addressed in the setting of the control parameters (i.e. matrices $R$ and $W$ ), as well as in the setting of the reference glucose trajectory, realizing a tradeoff between closed-loop fast asymptotic stability (suitably negative real part eigenvalues) and transient behavior (possibly smooth trajectories without oscillations). Simulations reported in [30] (see Figs. 2 and 3) show that the issue to prevent the switching off of the regulator cannot be ensured, at least when implementing the control law on a virtual environment, accounting for uncertainties affecting sensors and actuators devices as well as model parameter uncertainties; nevertheless, undesired oscillations can successfully be avoided.

\subsection{Evaluation criteria and validation}

Numerical simulations run by closing the control loop on (1) allowed to set the control parameters in order to track safely the desired glycemia within a couple of hour of insulin administration (see [25] for the details). Of course a realistic validation requires to build up an environmental framework which comprises measurement uncertainties, insulin pump malfunctioning, intra-patient variability as well as the discretization of the algorithm, because of real-time devices involving digital glucose sensors and insulin pumps: the former provide quite reliable measurements of plasma glycemia at given sample times, whose frequency is limited by the time needed to analyze plasma glucose on a bed-side analyzer, [3]; the latter are used to administer insulin by means of piecewise-constant infusions. To this end simulations have been carried out by discretizing the proposed control law at suitable sampling period $\Delta$, according to the following scheme $(k=0,1, \ldots)$ : 
1. at time $k \Delta$ the measurement of $G(k \Delta)$ is delivered by the sensor;

2. at time $k \Delta$ the control input is computed by means of the available state estimates $\widehat{G}(k \Delta), \widehat{G}\left(k \Delta-\tau_{g}\right), \widehat{I}(k \Delta)$, see (16):

$$
\frac{u(k \Delta)}{V_{I}}=\frac{S\left(\widehat{G}(k \Delta), \widehat{I}(k \Delta), \widehat{G}\left(k \Delta-\tau_{g}\right)\right)-v(k \Delta)}{K_{x g i} \widehat{G}(k \Delta)} ;
$$

if $u(k \Delta)<0$, then $u(k \Delta)$ is forced to be 0 ;

3. the constant infusion $u(k \Delta)$ is administered to the patient in the time interval $[k \Delta,(k+1) \Delta)$;

4. simultaneously with item [3.], the controller device integrates numerically the following equation, see (12), in the time interval $[k \Delta,(k+1) \Delta)$, using the available measurement $G(k \Delta)$ and past estimations:

$$
\begin{aligned}
& {\left[\begin{array}{l}
d \widehat{G} / d t \\
d \widehat{I} / d t
\end{array}\right]=\left[\begin{array}{c}
-K_{x g i} \widehat{G}(t) \widehat{I}(t)+\frac{T_{g h}}{V_{G}} \\
-K_{x i} \widehat{I}(t)+\frac{T_{i G \max }}{V_{I}} f\left(\widehat{G}\left(t-\tau_{g}\right)\right)+\frac{u(k \Delta)}{V_{I}}
\end{array}\right]} \\
& +Q^{-1}(\widehat{G}(t), \widehat{I}(t)) W(G(k \Delta)-\widehat{G}(k \Delta)) ;
\end{aligned}
$$

5. the value of $k$ is incremented by 1 .

Notice that (20) provides the state estimation in the prediction interval $[k \Delta,(k+$ 1) $\Delta)$. A crucial point in validating the synthesized control law has been to exploit two distinct models of the glucose-insulin system [30]. The minimal model (1), easy-to-handle from the control perspective, though physiologically meaningful and mathematically coherent, is exploited to synthesize the control law. A different model, more comprehensive, multi-compartmental, is exploited to simulate a Virtual Patient onto close the artificial pancreas. To this end, the model published in [6], recently accepted by the Food and Drug Administration as a substitute of animal trials [15], has been considered. The idea is sketched in Fig.1. The Virtual Patient stands for the chosen model for validation (and, in a hopefully not too far future, could be replaced by a real patient). The model identification dashed arrow pointing to the DDE model-based controller from the Virtual Patient block refers to a methodology to identify the DDE model parameters on the basis of a chosen virtual procedure (for instance, in [30] we considered a virtual IVGTT). The control parameter setting dashed arrow working on the DDE model-based controller block stands for the setting of the control parameters (i.e. matrices $R$ and $W$ ), a procedure achieved by means of trial and error simulations run on the DDE model without assuming failures or malfunctioning. The solid arrows refer to the closed loop system, which is implemented once the aforementioned preliminary two tasks have been properly fulfilled: the Virtual Patient provides sampled glucose measurements to the controller, and the DDE model-based controller provides the proper insulin infusion rate to be applied in the time interval $[k \Delta,(k+1) \Delta)$.

Moreover, in [30] the control parameters have been set once and for all the subjects of a population generated by sampling the many parameters of the virtual pa- 


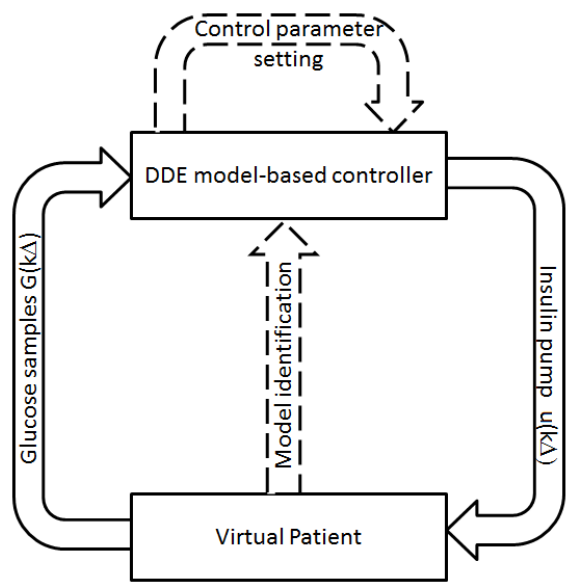

Fig. 1 Sketch of the virtual environment built up to validate the model-based control law.

tient provided by [6], each with a 5\% of Coefficient of Variation (CV). Moreover, glucose measurements are supposed to be affected by a $5 \%$ of $\mathrm{CV}$ as well as the insulin infusion is supposed to be affected by a $15 \%$ of CV.

Safety and efficacy criteria have been set according to the ones reported in [3]. The application of these criteria to a population of VPs, with an average basal glycemia of $9 \mathrm{mM}$, provides very interesting results. In case of absence of meals, no disturbance signal $d(t)$ in (1), on a population of $10,000 \mathrm{VPs}$, no hypoglycemia cases are reported (glycemia never reduces below $3.3 \mathrm{mM}$ ), in favor of very good efficacy results: more than $99.8 \%$ of diabetic VPs definitely reduce glycemia below $7 \mathrm{mM}$ within the first 3 hours of simulated experiments. These results are shown to be robust with respect to a discretization period $\Delta \in[5,15] \mathrm{min}$. Analogous very good results are obtained by applying the same control law on a $24 \mathrm{~h}$ temporal period, accounting for the administration of three meals (treated as unknown disturbances). Indeed, besides the complete absence of hypoglycemia cases, glycemia is constrained below $11 \mathrm{mM}$ within the 2 hours from the meal administration and during the period before the successive meal, for a set of diabetic VPs which exceeds 95\% of the population (see [30] for the details).

Remark 2. It is well known that disturbances like meals are hard to anticipate in timing, in amount and in the rate of effective absorption of the nutrient. By treating the meal $d(t)$ in (1) as a completely unknown disturbance, it is shown in [26] that the aforementioned closed-loop system (1), (12), (16-18) enjoys the local Input-to-State Stability property with respect to the unknown disturbance $d(t)$.

Remark 3. Copying with the need of an implementable discrete-time control law, a different philosophy would be to work on a discretized glucose-insulin system and then synthesize the control law according to the discrete framework. In [23] the DDE model in (1) has been discretized according to [1] and the digital control law is 
designed according to [34] where the dummy output proposed in [1] is used, in order to preserve under sampling the full relative degree of the continuous time model, up to an approximation of order 3 in the sampling period. The digital control law proposed in [23] guarantees the asymptotic stability of the suitably approximated sampled glucose insulin system.

\section{Observer-based control by means of subcutaneous insulin infusion}

Glucose control strategies actuated by means of subcutaneous insulin administration (see [2] and references therein) are easier to realize and, indeed, they can be managed nowadays by the patients themselves. However, in order to design closedloop control strategies, the insulin absorption from the subcutaneous depot needs also to be considered (see, among the others [11, 16, 21]). An excellent review of the available models presently adopted for blood glucose regulation as well as the closed-loop control methodologies and technical devices (blood glucose sensors and insulin pumps) may be found in [4] and references therein.

In order to synthesize a subcutaneously delivered insulin therapy, the modelbased approach requires to endow the glucose-insulin DDE model (1) with the subcutaneous insulin absorption compartment, see the complete model (3). The goal is to achieve a desired euglycemic glucose level for patients with a basal hyperglycemic state. No exogenous glucose disturbances are here considered (i.e. $d(t)=0$ in (3)).

According to [24, 28], by applying the theory of exact input-output feedback linearization with delay cancellation (see $[8,10,18,19])$, with respect to the input $u(t)$ and the output $G(t)-G_{d}\left(G_{d}\right.$ is the desired level of glycemia), the following control law is found:

$$
u(t)=V_{I} t_{\max , I}^{2} \frac{\alpha(\cdot)-v(t)}{K_{x g i} G(t)},
$$

where $\alpha(\cdot)$ is a function of the system variables at the present time $G(t), I(t), S_{1}(t)$, $S_{2}(t)$, and of some of them at delayed times (namely of $G\left(t-\tau_{g}\right), I\left(t-\tau_{g}\right), S_{2}(t-$ $\tau_{g}$ ) and $G\left(t-2 \tau_{g}\right)$ ), see [24] for the explicit formulation. By applying (21) to (3), it comes that the closed-loop system may be written by using the new variables $z(t)=\left[\begin{array}{llll}G(t) & G^{(1)}(t) & G^{(2)}(t) & G^{(3)}(t)\end{array}\right]^{T}$ as:

$$
\dot{z}(t)=A_{b} z(t)+B_{b} v(t), \quad t \geq 0,
$$

with $A_{b}, B_{b}$ the fourth-order Brunowski pair and the brackets ${ }^{(i)}$ denoting the $i$-th time derivative. By setting the outer input $v(t)=\Gamma e(t)$, with

$$
e(t)=z(t)-z_{d}, \quad z_{d}=\left[\begin{array}{llll}
G_{d} & 0 & 0 & 0
\end{array}\right]^{T},
$$

the error dynamics becomes: 
Title Suppressed Due to Excessive Length

$$
\dot{e}(t)=H e(t), \quad H=A_{b}+B_{b} \Gamma .
$$

Since the Brunowski pair is controllable, we design the gain matrix $\Gamma \in \mathbb{R}^{1 \times 4}$ in order to make Hurwitz $H$ and, therefore, the tracking error between plasma glycemia and its reference signal (the first component of $e(t)$ ) converges exponentially to zero.

Besides the drawback of real-time insulin measurements involved in the aforementioned glucose control law (shared also by the intra-venous insulin delivery therapies dealts with in Section 3), here also subcutaneous insulin measurements are required, which are quite impossible to obtain, especially in a real-time closed-loop framework. In order to overcome such problems, in [21] a state observer for system (3) is considered, with the aim of estimating the insulin on the basis of continuous time glucose measurements.

In order to design the state observer for (3), define $X(t)=\left[G(t) I(t) S_{2}(t) S_{1}(t)\right]^{T} \in$ $\mathbb{R}^{4}$ such that the DDE system (3) with $d(t)=0$ can be formally written in the more compact form:

$$
\dot{X}(t)=\mathscr{F}\left(X(t), X\left(t-\tau_{g}\right)\right)+B_{b} u(t),
$$

where $\mathscr{F}: \mathbb{R}^{4} \times \mathbb{R}^{4} \mapsto \mathbb{R}^{4}$ is defined for $X=\left[X_{1} X_{2} X_{3} X_{4}\right]^{T}, Y=\left[Y_{1} Y_{2} Y_{3} Y_{4}\right]^{T} \in \mathbb{R}^{4}$ as:

$$
\mathscr{F}(X, Y)=\left(\begin{array}{c}
-K_{x g i} X_{1} X_{2}+\frac{T_{g h}}{V_{G}} \\
-K_{x i} X_{2}+\frac{T_{i \max }}{V_{I}} f\left(Y_{1}\right)+\frac{1}{t_{\max , I}} X_{3}, \\
\frac{1}{t_{\max , I}} X_{4}-\frac{1}{t_{\max , I}} X_{3} \\
-\frac{1}{t_{\max , I}} X_{4}
\end{array}\right)
$$

The measured output is, then, given by $y(t)=G(t)=C_{b} X(t)$, where $C_{b}=$ $\left[\begin{array}{llll}1 & 0 & 0 & 0\end{array}\right]$.

The observer for system (25-26) adopted in [21] is the one developed in [9], given by the following neutral system, with $\widehat{X}(t), w(t) \in \mathbb{R}^{4}$ :

$$
\dot{\hat{X}}(t)=\mathscr{F}\left(\widehat{X}(t), \widehat{X}\left(t-\tau_{g}\right)\right)+B_{b} u(t)+w(t), \quad t \geq 0,
$$

with

$$
w(t)=Q^{-1}\left(\widehat{X}(t), \widehat{X}\left(t-\tau_{g}\right)\right)\left(W\left(y(t)-C_{b} \widehat{X}(t)\right)-Q_{1}\left(\widehat{X}(t), \widehat{X}\left(t-\tau_{g}\right)\right) w\left(t-\tau_{g}\right)\right) .
$$

Matrices

$$
\begin{aligned}
& Q\left(\widehat{X}(t), \widehat{X}\left(t-\tau_{g}\right)\right)=\frac{\partial \Theta\left(\widehat{X}(t), \widehat{X}\left(t-\tau_{g}\right)\right)}{\partial \widehat{X}(t)} \\
& Q_{1}\left(\widehat{X}(t), \widehat{X}\left(t-\tau_{g}\right)\right)=\frac{\partial \Theta\left(\widehat{X}(t), \widehat{X}\left(t-\tau_{g}\right)\right)}{\partial \widehat{X}\left(t-\tau_{g}\right)}
\end{aligned}
$$

are obtained from the partial derivatives of the function $\Theta(\cdot, \cdot)$ (see [9]), which is formally defined as the aggregate of the output $G(t)$ and its first 3 time derivatives, obtained according to (3). $\Theta(\cdot, \cdot)$ is a function of the system variables at the present time (i.e. $\left.G(t), I(t), S_{1}(t), S_{2}(t)\right)$ and of some of them at the delayed time (namely $G\left(t-\tau_{g}\right)$ and $I\left(t-\tau_{g}\right)$ ) (see [21] for the explicit expression of $\Theta(\cdot, \cdot)$ ). 
When the function $\Theta$ is used for computations in (29), $\widehat{X}(t)$ takes the place of $\left[G(t) I(t) S_{2}(t) S_{1}(t)\right]^{T}$, for any required time $t$. The gain matrix $W \in \mathbb{R}^{4 \times 1}$ is chosen such that the matrix $\widehat{H}=A_{b}-W C_{b}$ is Hurwitz.

It has been shown in [9] that the gain matrix $W$ can be properly designed to ensure the global asymptotic convergence to zero of the observation error, provided that proper conditions are satisfied. Such conditions are not completely satisfied by the system at hand (for instance, the functions involved in (3) are not globally Lipschitz). However, we are not interested in the convergence of the observation error to zero for any initial state and input signal in bounded sets, as in [9], but only in the convergence, at least locally, of the state variables of the closed-loop system to the desired equilibrium. To this end, we exploit the observer equations to close the loop from the observed state $\widehat{X}(t)$, so that the state variables in (21) are replaced by their estimates. Then, the control law becomes:

$$
u(t)=V_{I} t_{\max , I}^{2} \frac{\alpha(\hat{*})-v(t)}{K_{x g i} C_{b} \widehat{X}(t)}, \quad v(t)=\Gamma\left(\hat{z}(t)-z_{d}\right),
$$

with $\hat{z}(t)=\Theta\left(\widehat{X}(t), \widehat{X}\left(t-\tau_{g}\right)\right.$ and $\alpha(\hat{*})$ denoting the function $\alpha(\cdot)$ computed in the observed state variables $\widehat{X}(t), \widehat{X}\left(t-\tau_{g}\right), \widehat{X}\left(t-2 \tau_{g}\right)$, instead of the real ones $X(t)$, $X\left(t-\tau_{g}\right), X\left(t-2 \tau_{g}\right)$.

The main result provided by [21] is that there exist matrices $W$ and $\Gamma$ such that, as long as the initial estimation error and the initial tracking error are sufficiently small, the evolution of the closed-loop system (25)-(30) asymptotically converges to $\mathscr{X}_{E}=\left[\begin{array}{ll}X_{E}^{T} & X_{E}^{T}\end{array}\right] \in \mathbb{R}^{8}$, where $X_{E}=\left[\begin{array}{llll}G_{d} & I_{d} & S_{2, d} & S_{1, d}\end{array}\right]^{T}$ is the equilibrium point of (3) when the control input $u(t)=u_{d}=S_{1, d} / t_{\max , I}$ (see next remark) is provided.

Remark 4 . The constant terms $G_{d}, I_{d}, S_{2, d}, S_{1, d}$ are such that, if at a given time instant $\bar{t} \geq 0$, it is $G(\tau)=G_{d}, I(\tau)=I_{d}, S_{2}(\tau)=S_{2, d}, S_{1}(\tau)=S_{1, d}$, for $\tau \in\left[\bar{t}-2 \tau_{g}, \bar{t}\right]$, and the control law $u(t)$ designed as in (21) is applied, then the solution of (3) with $d(t)=0$ is $G(t)=G_{d}, I(t)=I_{d}, S_{2}(t)=S_{2, d}, S_{1}(t)=S_{1, d}$, for $t \geq \bar{t}$, and the control law becomes $u(t)=u_{d}, t \geq \bar{t}$. In other words, once $G_{d}$ has been chosen, we may compute $I_{d}, S_{2, d}, S_{1, d}$ and $u_{d}$ as the reference levels of the system variables and of the control input that asymptotically correspond to a perfect tracking of $G_{d}$. As a matter of fact, the state $X_{E}=\left[\begin{array}{llll}G_{d} & I_{d} & S_{2, d} & S_{1, d}\end{array}\right]^{T} \in \mathbb{R}^{4}$ is the equilibrium point of the closed loop system (3)-(21).

\section{Conclusions}

The common denominator of the proposed insulin administration therapies are given by (i) the model-based approach, (ii) the use of a state observer to predict real-time insulin measurements. Indeed, only glucose measurements have been considered, with insulin administered both intravenously and subcutaneously. The resulting control laws are theoretically appealing and, at least for the intravenous case, robust with respect to many sources of uncertainties, measurements errors and actuator 
malfunctioning, according to a virtual environment making use of a different and more comprehensive model of the glucose-insulin system. The same philosophy has been recently applied also to a different clinical framework such as the one of the Euglycemic Hyperinsulinemic Clamp (EHC) [27, 29], a nontrivial perturbation experiment during which large amounts of insulin are administered intra-venously to the subject, and exogenous glucose is administered, according to given protocols, in order to keep glycemia constant. In this chapter we have briefly surveyed the recent results about estimation methods for the glucose-insulin system by means of state observers for time-delay systems, in perspective with the control problem.

\section{References}

1. Barbot, J.P., Monaco, S., Normand-Cyrot, D.: A sampled normal form for feedback linearization. Math Control Signal Syst. 9, 162188 (1996)

2. Bellazzi, R., Nucci, G., Cobelli, C.: The subcutaneous route to insulin dependent diabetes therapy, IEEE Engineering in Medicine and Biology. 20, 54-64 (2001)

3. Chassin, L.J., Wilinska, M.E., Hovorka, R.: Evaluation of glucose controllers in virtual environment: methodology and sample application. Artif Intell Med. 32, 171-181, (2004)

4. Chee, F., Fernando, T.: Closed loop control of blood glucose. In Thoma, M., Morari, M. (eds.) Lecture Notes in Control and Information Sciences, Springer-Verlag Berlin, Heidelberg (2007)

5. Chee, F., Savkin, A.V., Fernando, T.L., Nahavandi, S.: Optimal $H_{\infty}$ insulin injection control for blood glucose regulation in diabetic patients. IEEE Trans Biomed Eng. 52, 1625-1631 (2005)

6. Dalla Man, C., Rizza R.A., Cobelli, C.: Meal simulation model of the glucose-insulin system. IEEE Trans Biomed Eng. 54, 1740-1749 (2007)

7. Dua, P., Doyle, F.J., Pistikopoulos, E.N.: Model-based blood glucose control for Type 1 diabetes via parametric programming. IEEE Trans Biomed Eng. 53, 1478-1491 (2006)

8. Germani, A., Manes, C., Pepe, P.: Local asymptotic stability for nonlinear state feedback delay systems. Kybernetika. 36, 31-42 (2000)

9. A. Germani, C. Manes, P. Pepe, An asymptotic state observer for a class of nonlinear delay systems, Kybernetika, 37(4), pp.459-478, 2001.

10. Germani, A., Manes, C., Pepe, P.: Input-output linearization with delay cancellation for nonlinear delay systems: the problem of the internal stability. Int J Robust Nonlinear Control. 13(9), 909-937 (2003)

11. Hovorka, R., Canonico, V., Chassin, L.J., Haueter, U., Massi-Benedetti, M., Federici, M.O., Pieber, T.R., Schaller, H.C., Schaupp, L., Vering, T., Wilinska, M.E.: Nonlinear model predictive control of glucose concentration in subjects with type I diabetes. Physiol Meas. 25, 905-920 (2004)

12. Kong, J.D., Kumar, S.S., Palumbo, P.: DDE models of the glucose-insulin system: a useful tool for the artificial pancreas. In Delitala, M., Ajomne-Marsan, G. (eds.) Managing Complexity, Reducing Perplexity in Biological Systems, Springer Proceedings in Mathematics \& Statistics. 67, pp.109-117 (2014)

13. Kovàcs, L., Benyò, B., Bokor, J., Benyò, Z.: Induced L2-norm minimization of glucoseinsulin system for Type I diabetic patients. Comput Meth Programs Biomed. 102, 105-118 (2011)

14. Kovàcs, L., Kulcsàr, B., Gyŏrgy, A., Benyò, Z.: Robust servo control of a novel type 1 diabetic model. Optim Control Appl Methods. 32, 215-238 (2011)

15. Kovatchev, B.P., Breton, M.D., Dalla Man, C., Cobelli, C.: In silico model and computer simulation environment approximating the human glucose/insulin utilization. Food and Drug Administration Master File MAF 1521 (2008) 
16. Magni, L., Raimondo, D.M., Dalla Man, C., De Nicolao, G., Kovatchev, B., Cobelli, C.: Model predictive control of glucose concentration in type I diabetic patients: an in silico trial. Biomed Signal Process Control, 4, 338-346 (2009)

17. Makroglou, A., Li, J., Kuang, Y.: Mathematical models and software tools for the glucoseinsulin regulatory system and diabetes: an overview. Appl Numer Math. 56, 559-573 (2006)

18. Marquez-Martinez, L.A., Moog, C.H. : Input-output feedback linearization of time-delay systems. IEEE Trans Autom Control. 49(5), 781-785 (2004)

19. Oguchi, T., Watanabe, A., Nakamizo, T.: Input-output linearization of retarded nonlinear systems by using an extension of Lie derivative. Int J Control. 75(8), 582-590 (2002)

20. Palumbo, P., Panunzi, S., De Gaetano, A.: Qualitative behavior of a family of delay differential models of the glucose insulin system. Discrete Contin Dyn Syst-Ser B. 7, 399-424 (2007)

21. Palumbo, P., Pepe, P., Kong, J.D., Kumar, S.S., Panunzi, S., De Gaetano, A.: Regulation of the human plasma glycemia by means of glucose measurements and subcutaneous insulin administration. 3rd IFAC International Conference on Intelligent Control and Automation Science (ICONS13), 96-101, Chengdu, China (2013)

22. Palumbo, P., Pepe, P., Panunzi, S., De Gaetano, A.: Robust closed-loop control of plasma glycemia: a discrete-delay model approach. Discrete Contin Dyn Syst-Ser B, 12(2), 455-468 (2009)

23. Palumbo, P., Pepe, P., Panunzi, S., De Gaetano, A.: Digital closed-loop control of plasma glycemia. 49th IEEE Conference on Decision and Control (CDC10), 833-838, Atlanta, Georgia (2010)

24. Palumbo, P., Pepe, P., Panunzi, S., De Gaetano, A.: Glucose control by subcutaneous insulin administration: a DDE modelling approach. 18th IFAC World Congress on Automatic Control (IFAC2011). pp.1471-1476, Milan, Italy (2011)

25. Palumbo, P., Pepe, P., Panunzi, S., De Gaetano, A.: Time-delay model-based control of the glucose-insulin system, by means of a state observer. Eur J Control. 6, 591-606 (2012)

26. Palumbo, P., Pepe, P., Panunzi, S., De Gaetano, A.: Observer-based closed-loop control for the glucose-insulin system: local Input-to-State Stability with respect to unknown meal disturbances, American Control Conference (ACC13), pp.1751-1756, Washington, DC (2013)

27. Palumbo, P., Pepe, P., Panunzi, S., De Gaetano, A.: Closed-loop glucose control: application to the Euglycemic Hyperinsulinemic Clamp. 52nd IEEE Conference on Decision and Control (CDC13). pp.4461-4466, Florence, Italy (2013)

28. Palumbo, P., Pepe, P., Panunzi, S., De Gaetano, A.: DDE model-based control of glycemia via sub-cutaneous insulin administration. In Vyhlídal, T., Lafay, J.-F., Sipahi, R. (eds.) Delay Systems. From Theory to Numerics and Applications. Advances in Delays and Dynamics, Springer International Publishing, 1, pp.229-240 (2014)

29. Palumbo, P., Pizzichelli, G., Panunzi, S., Pepe, De Gaetano, A.: Closed-loop control scheme for the Euglycemic Hyperinsulinemic Clamp: validation on virtual patients. IFAC World congress on Automatic Control (IFAC2014). pp.2088-2093, Cape Town, South Africa (2014)

30. Palumbo, P., Pizzichelli, G., Panunzi, S., Pepe, P., De Gaetano, A.: Model-based control of plasma glycemia: Tests on populations of virtual patients. Math Biosci. 257, 2-10 (2014)

31. Panunzi, S., De Gaetano, A., Mingrone, G.: Advantages of the single delay model for the assessment of insulin sensitivity from the intravenous glucose tolerance test. Theor Biol Med Model. 7(9) (2010)

32. Panunzi, S., Palumbo, P., De Gaetano, A.: A discrete single delay model for the Intra-Venous Glucose Tolerance Test. Theor Biol Med Model. 4(35) (2007)

33. Parker, R.S., Doyle III, F.J., Ward, J.H., Peppas, N.A.: Robust $H_{\infty}$ glucose control in diabetes using a physiological model. AIChE J. 46, 2537-2549 (2000)

34. Pepe, P.: Preservation of the full relative degree for a class of delay systems under sampling. J Dyn Syst Meas Control-Trans ASME. 125(2), 267-270 (2003)

35. Puckett, W.R., Lightfoot, E.N.: A model for multiple subcutaneous insulin injections developed from individual diabetic patient data. Am J Physiol-Endocrinol Metab. 269, E1115E1124 (1995) 
36. RuizVelázquez, E., Femat, R., CamposDelgado, D.U.: Blood glucose control for type I diabetes mellitus: a robust $H^{\infty}$ tracking problem. Control Eng Practice. 12, 1179-1195 (2004)

37. Van den Berghe, G.: Insulin therapy for the critically ill patient. Clinical Cornerstone. 5(2), 56-63 (2003)

38. Wild, S., Roglic, G., Green, A., Sicree, R., King, H.: Global prevalence of diabetes - Estimates for the year 2000 and projections for 2030. Diabetes Care 27(5), 1047-1053 (2004)

39. Wilinska, M.E., Chassin, L.J., Schaller, H.C., Schaupp, L., Pieber, T.R., Hovorka, R.: Insulin kinetics in Type-1 diabetes: continuous and bolus delivery of rapid acting insulin. IEEE Trans Biomed Eng. 52, 3-12 (2005) 